\title{
RECENT ADVANCES IN THE CONTROL OF BANCROFTIAN FILARIASIS
}

\author{
by \\ Manabu Sasa \\ Director, and Professer of Parasitology, the Institute of Medical Sciencs, \\ The University of Tokyo, Japan.
}

\begin{abstract}
:
Human flariasis due to Wuchereria Bancrofti is a disease endemic widely in tropical and subtropical regions of the World, and nearly ten thousand million of people in various countries, especially in Asia, are estimated to be residing in the areas where the disease is endemic. Furthermore, the disease is considered to be increasing in many countries along with the trend of urbanization.

In Japan, a number of endemic areas have been recorded from the man and some adjacent jslands, with extremely high incidences especially in the Amami and the Ryukyu lslands, Southern Japan. A country-wide filariasis control program was initiated in 1962, based on basic studies and pilot experiments carried out by research workers in a filariasis control study group for about ten years prior to the control project. The program starts with the survey and disignation of the endemic areas to becontrolled, and the microfilarial examination of all the people in the target areas are aimed to be carried out at least once a year. The drug, diethvlcarbamazine, is administered to all the posilive cases at the daily dose of $6 \mathrm{mg}$ per $\mathrm{kg}$ of the body weight for 12 successive davs. The control of vector mosquito, usualiy the Culex pipiens complex, is also applied to these areas, by both environmental sanitation and the use of insecticides. As the results, remarkable reductions in the rates of microfilarial carriers and the clinical cases have been achizved in all the areas under the program.
\end{abstract}

The author has been engaged in the studies on filariasis and its vectors in Southeast Asian countries in cooperation with the looal workers. Among various experiences, cspecially interesting result have been obtained in the use of American poeciliid fresh water fisles as the agents of biological control of larvae of the vector mosquitoes in highly polluted waters.

In this paper, it is intended to make a brief review of recent advances in the studies on rilariasis and related diseases endemic in Southern Asia, with reference to the activity of international organizations, such as WHO, the Japan-United States Cooperative Micdical Science Program, and the Colombo Plan.**

**The main rext has, unfortunaiely, not reached us in time. Hence the full text may be published later. -Editor 
$\frac{\text { PUBLIC HEALTH REPORTS }}{\text { J. Nep. Med. Ass. (1971) } 9.1--2}$

\section{SMALL-POX INCIDENCE}

by

DR. P.N. Shrestha, M.B.,B.S, M.P.H (U.S.A)

Project-In-Charge

Small-Pox Cotrol Pilot Project

His Majesty's Govt. of Nepal

Introduction:

Since the beginning of the eradication program, the incidence of smallpox in Nepal has been declining steadily. The number of reported cases for 1968, 1969 and 1970 were 249 , 163 and 78 respectively. For more than 3 years, smallpox has been restricted to the Terai region only and except for a single unconfirmed case in Syangja in 1969, there has been no. other case in the bills. There has been no case in Kathmandu Valley for more than 4 years.

Seasonal Pattern:

No seasonal pattern for the incidence of smallpox has been observed (Fig. 1). During

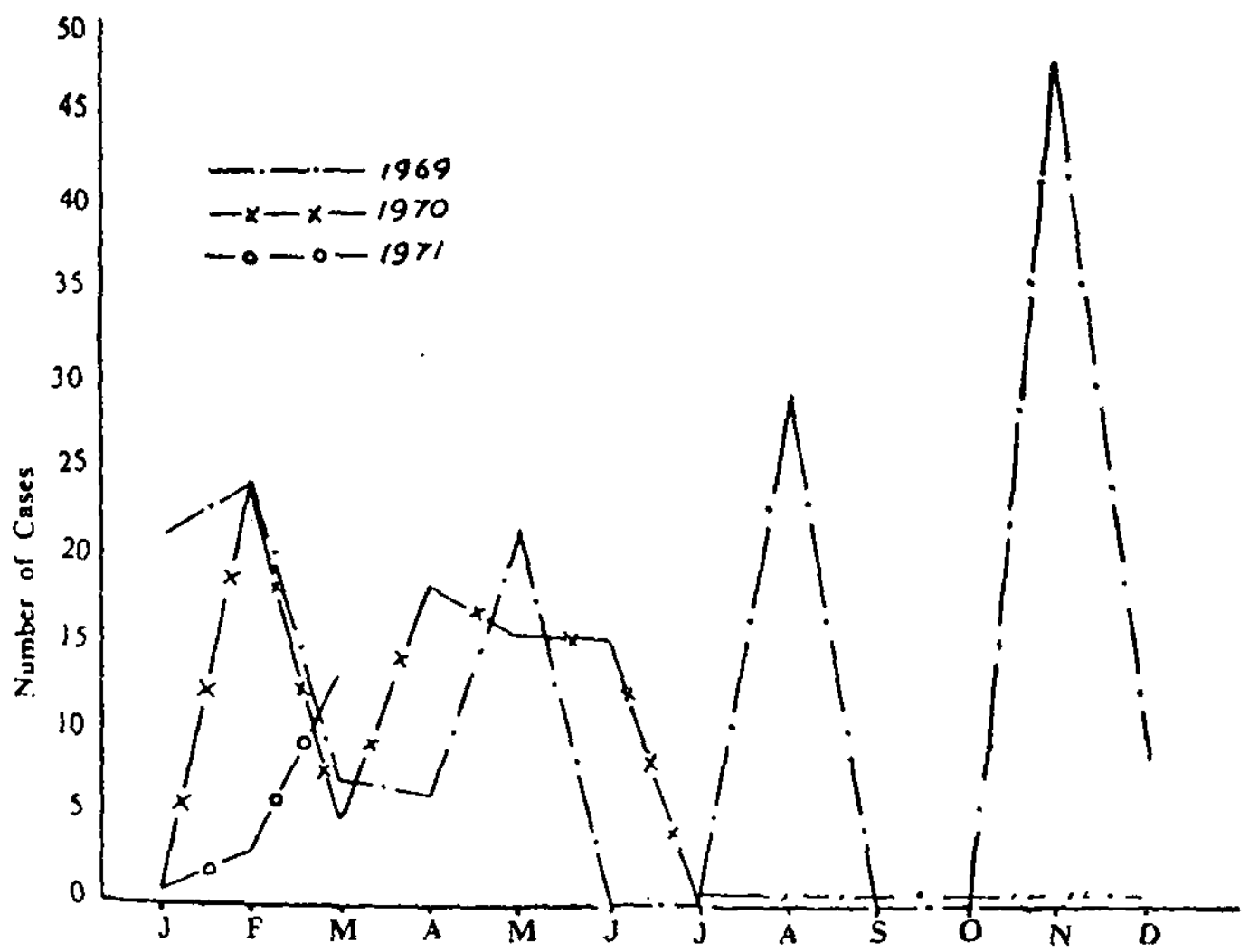

Fig 1. Smallpox Incideace 
the first 3 months of 1971,17 cases have been reported to the Smallpox Eradication Project Headquarter (SFP HQ) and the corresponding figures for 1969 and 1970 were 52 and 30 respectively.

Outbreak in 1971:

On March 22, 1971 an outbreak of smallpox in Karmaiya Panchayat of Sarlahi District, Janakpur Zone was reported to the SEP HQ by its district office. Investigation was immediatly undertaken. It was found that the index case was an 8 year old girl of neighboring Chhauria Villaye in India who came to Karmaiya on 22 Jan. 1971. She had fever soon after arrival and developed rash on $26 \mathrm{Jan}$. During the next two months the infection spread to others through 4 generation of disease transmissions. (Fig.2).

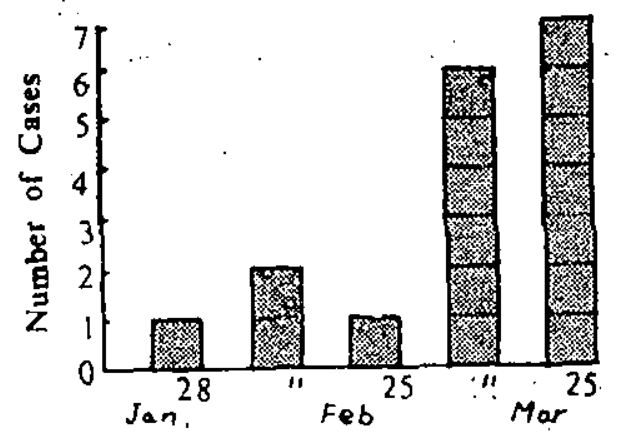

Fig 2 Sinallpox by week of onset

There were in all 17 cases with 9 deaths (Table 1). Of these 4 adults, one of whom had also been vaccinated; had variolation scars. A 12 year old girl who died was reported to have been vaccinated 2 years ago but it is not known whether the vaccination was successful or not. One 1 t year old toy who was vaccinated successfully on $17 \mathrm{March}$, developed rash on 25 March and survived. The vaccination status of the index case who returnet back to India was not known.

Table 1.

Cases by Age Group and Vaccination Status

Age group

Under $1 \mathrm{yr}$

$1-4$ ye.

$5-14 \mathrm{yr}^{\circ}$

Above 14 yr

Total

$\begin{array}{ccccc}\text { Vaccinated" } & \text { Not vaccinated } & \text { Unknoun } & \text { Total } & \text { Number dead } \\ 0 & 5 & 1 & 6 & 5 \\ 1 & 1 & 0 & 2 & 1 \\ 0 & 0 & 2 & 2 & 1 \\ 4 & 3 & 0 & 7 & 2 \\ 5 & 9 & 3 & 17 & 9\end{array}$

- includes variolation successfully vaccinated during the outbreak 
SMNL-POX

The cotal case fatality rate (CFR) was $53 \%$ (Table 2). None of the vaccinated or variolated cases died. The CFR for non--vaccinated and unknown groups was $77.7 \%$ and $66.6 \%$ respectively. The mortality rate was highest in infants who formed the least protected group.

Table 2.

Case Fatality Rate (CFR)

No. of cases No. dead CFR $(\%)$

$\begin{array}{lccc}\text { Vaccillate } J^{e} & 5 & 0 & 0 \\ \text { Not vaccinated } & 9 & 7 & 77,7 \\ \text { Unknown } & 3 & 2 & 66.6 \\ \text { Total } & 17 & 9 & 53.0 \\ & & \text { e includes variolation }\end{array}$

Apart from the mass vaccination program for protection of a sizable proportion of the population. active surveillance and prompt reporting of any suspected case of smallpox by all sections of the particularly health personnel are necessary for the eradication of the disease rrom the country.

\section{BCG Immunizations}

BCG inoculations are being given us a mass campaign in the districts of Kathmandu Valley, Kabhrepalancok and Koshi Zone (Table 3). In addition $\mathrm{MCH}$ clinics and public health programs of a few hospitals are also immunizing children against tuberculosis in other districts. The total number of inoculations during the first 3 months of 1971 was 31059 .

Table 3.

BCG Inoculations by Districts

\begin{tabular}{lrrrrr}
\multicolumn{1}{c}{ District } & Under 1 yr & $1-4$ yr & $5-14$ yr & Above 14 yr & Total \\
Kath. Valley & 175 & 186 & 57 & 1 & 959 \\
Kabhrepalanchok & 765 & 2434 & 5327 & 131 & 8657 \\
Koshi Zone & 2074 & 5487 & 11562 & 1211 & 20234 \\
Others & 406 & 337 & 442 & 24 & 1209 \\
Total & 3960 & 8344 & 17388 & 1367 & 31059
\end{tabular}

These inoculations were mostly given by Tuberuclosis Control Pilot Project, Koshi Zonal Project, MCH clinics and Maternity hospitals (table 4). BCG is given direct to all children under 15 years including newborn babies in maternity wards of the hospitals. Among the inoculees, $5 .-14$ yr. form the biggest group (56\%) followed by $1--4$ yr $(26.8 \% 0)$ and under 1 yr $(12.7 \%)$. 
Table 4.

BCG Inonculations by Project

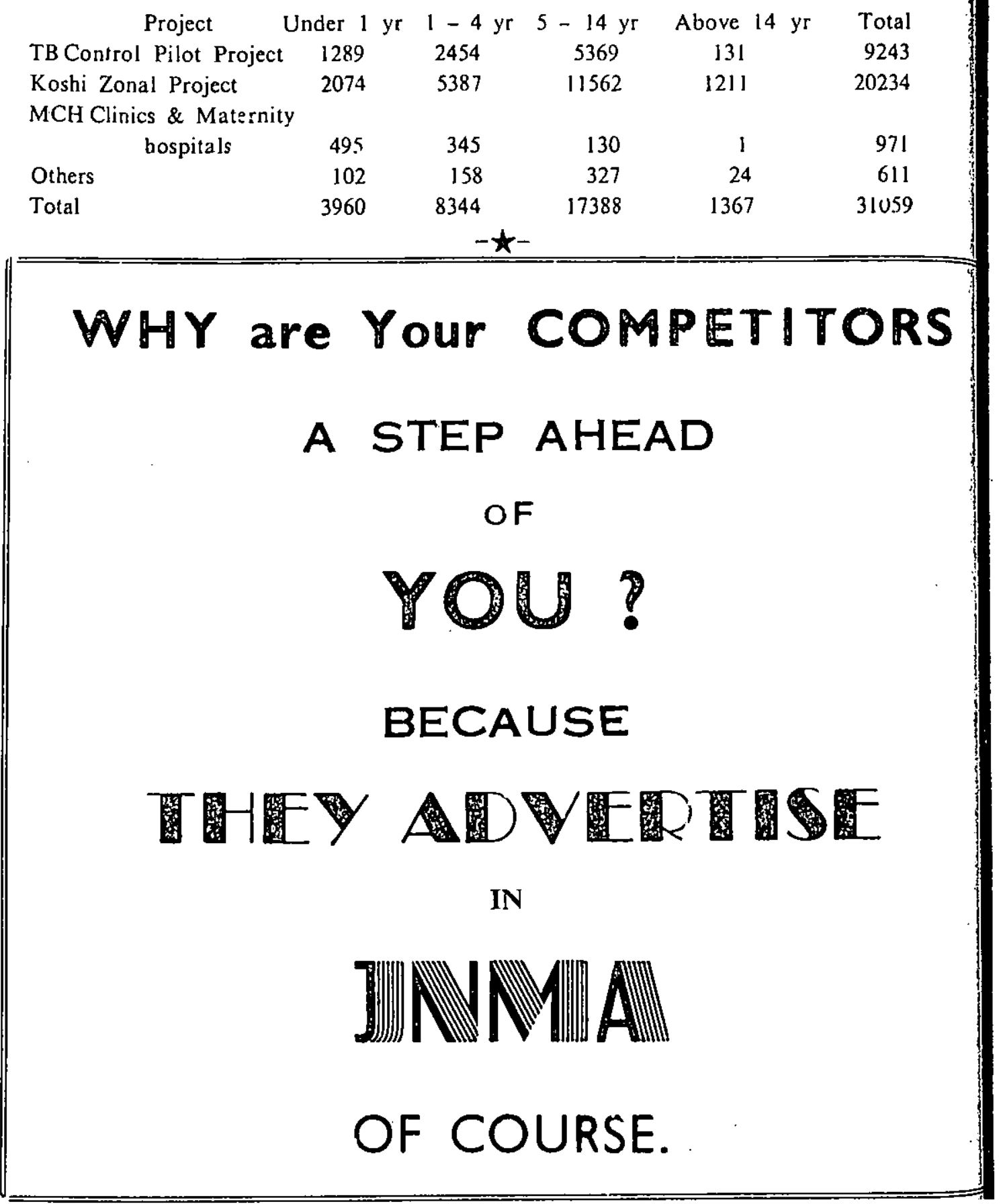

